\title{
A Comment on the Future Perspectives of Health Insurance in the USA
}

\author{
by John K. Kittredge*
}

First of all, we should recognize that the United States does not have a governmental health program covering all or even most of its citizens. In the United States, health care costs for the population under 65 are borne primarily by private financing.

Private health insurance in the United States really started in the 1930's with the formation of the first of the Blue Cross organizations. Its motivation was less to provide protection for those who are insured than it was to help the financial stability of the hospitals whose bills were to be paid. Private health insurance did not start spreading widely until the 1940's.

But the decade of most rapid expansion of private health insurance was the 1950's. That period set a pattern of provision of health insurance primarily through employer-sponsored plans. As time went on, employers tended to pay an increasing share of the cost. The 1950's and 1960's also saw a considerable broadening of the kinds of medical costs covered by private health insurance. Although they also saw an expansion of coverage under policies purchased by individuals, by far the greatest coverage has been through employment.

In the mid-1960's, our only governmental health insurance programs, other than for Federal employees, were adopted-Medicare, covering individuals age 65 and over, and Medicaid, providing coverage for some of the poors.

All of the coverage which I have described provided benefits by paying for the medical care services which were obtained by, and provided to, the individuals insured. Each individual hospital stay, diagnostic test, physician's visit, etc., was paid for. The plans provided relatively little in the way of financial controls other than to require that the service be "medically necessary" and that the charge for the service be "reasonable". In the litigous American society, it is difficult to prove that a medical service was not necessary. It was somewhat less difficult to prove that the charge for the service was unreasonable. Most of the health insurance coverage still provided in the United States follows this pattern, but third party payers have gained greater control over the ability to determine medical necessity.

\footnotetext{
* Executive Vice President the Prudential Insurance Company of America; paper presented at the Geneva Association Assembly, June 16, 1987, Berlin.
} 
The first health maintenance organization, or HMO, dates back many years, but HMO's didn't begin to spread widely until the 1970's. Prudential first became involved in studying HMO's and our role in them in 1968. Our first active involvement with an HMO was managing one which had been started by others. Prudential started its first HMO in Houston, Texas, in 1975.

Some of the experience we and others had in developing and operating HMO's may be of interest and may be pertinent to looking at the applicability of our concepts in Europe.

The barriers which we encountered fall into two categories-those which dealt with the providers of medical care and those which dealt with our potential customers. The providers were, of course, primarily physicians and hospitals. The real key was physicians, however. The first step in developing an $\mathrm{HMO}$ is to attract physicians to join the organization. Physicians aren't much different from anyone else in their concern about developing and protecting their incomes. Our difficulties in attracting physicians initially were adversely affected by their ability to earn very good incomes without becoming part of an HMO. In addition, our difficulties were increased by the attitude of physicians, particularly those active in medical organizations, that there was something morally or ethically wrong with practicing medicine in a Health Maintenance Organization.

Hospitals were somewhat easier to deal with, particularly after physicians joined the HMO and there were expectations of additional hospital patients coming from those physicians. Hospitals, however, were quite reluctant to charge lower prices in the expectation of larger volumes of patients.

Much of what I have described has changed. In many areas of the country, we have a surplus of physicians. Physicians are concerned about obtaining enough patients to maintain their standards of living. Younger physicians also seem to be interested in joining organizations and not having to deal with the costs of starting a new practice or of running physicians' offices. In most areas of the country, it has become relatively easy to attract physicians to HMO's.

Similarly for hospitals, we have gone through a period during which the use of hospitals to treat illness has decreased. Occupancy of hospital beds has been decreasing as a percentage of the total beds available over the last few years. As a result, we have a very sizable surplus of hospital beds in the United States. Hospitals are concerned about finances and are increasingly willing to make price concessions to HMO's to attract patients.

Once physicians have been attracted to join HMO's, the next step is to influence changes in the ways in which they practice medicine. HMO's have accomplished this through management and oversight of the physicians practicing in the HMO's. An incentive is also provided if physicians are financially affected by the profits or losses of the HMO. In most HMO's today, some element of compensation for the physician staff is dependent upon the financial results of the HMO. 
Even with financial incentives to physicians to avoid unnecessary medical care, we have not infrequently had difficulties in changing patterns of medical practice from what they had been. Our experience has been that very conscious efforts must be made to change physicians' practice patterns.

Turning now to the barriers related to the customer: when we first became involved in HMO's, other than on the west coast, the entire concept of HMO's was relatively little known by employers or by employees. We soon learned that marketing of HMO coverage to replace the more traditional coverage first required education as to what an HMO is and what its advantages are. We also learned that marketing of HMO's is a two-step sale. We first had to sell the employer on offering HMO coverage and then we had to sell the employees on enrolling for it, since HMO coverage is almost always offered as an option to a traditional plan.

Since the 1970 's, the marketing barriers have dropped significantly. The concept and advantages of HMO's and similar systems is increasingly understood by employers. Employees are bombarded in many cities by advertising from competing HMO's and the concept has become much more familiar to them. Our major task now is to make the sale that the HMO chosen is the Prudential HMO rather than having to sell the HMO concept itself.

Over the last 15 years, there was been a distinct shift in type of HMO. The earliest HMO's were ones in which the physicians practice medicine together in one or more clinics. The physicians in these organizations usually spend all of their time treating HMO patients. The second type of HMO is one in which the physicians practice in their own offices and are not located together in a clinic.

The prevalence of the second type of $\mathrm{HMO}$ has been increasing rapidly in recent years. It avoids the costs of constructing buildings to be used as clinics. It also permits physicians to receive HMO patients without disrupting their existing practices. Physicians in the clinic model are typically paid a salary with an additional payment based upon the profitability of the HMO. Physicians in the other model typically receive a fee for each service they provide. The fee is lower than their normal fee for the sevice, but if the plan is profitable they receive additional compensation.

What have HMO's accomplished in controlling health care costs? The most significant change has been a reduction in the use of hospitals. In a typical HMO, patients will occupy hospital beds for 350 days per year for each 1,000 enrollees. This compares with hospital confinement rates which hovered around 800 days per 1,000 enrollees under traditional insurance plans in the 1970's. The hospital utilization for traditional plans has, however, been dropping as controls on hospital utilization have been tightened up and physicians have learned that they can practice in a way which is less dependent upon hospital confinement. Now a typical plan might use about 500 days of hospital confinement per 1,000 enrollees. 
The reduction in hospital use has come from a number of sources. Many surgical procedures previously performed while the patient was confined in a hospital are now being performed without any hospital confinement. Diagnostic tests are performed on an ambulatory basis where hospital confinement had been common previously. Even where hospital confinement is ultimately necessary, often the necessary diagnostic testing is done before the patient enters the hospital. In addition, where hospital confinement is required, confinements are shorter. As an example, in many parts of the country normal deliveries of children require only 2 or 3 days of hospital confinement rather than 5 , which was typical a few years ago.

There have also been some savings by reducing the use of specialists. In an HMO, each enrollee is assigned to, or chooses, a primary care physician. The primary care physician may be in family practice, internal medicine, or pediatrics. The primary care physician acts as a gatekeeper and directs the medical care of the member. Referral to specialists is made by the primary physician. In the traditional practice of medicine, individuals are free to refer themselves to specialists which frequently leads to unnecessarily expensive care.

The 1980's have been the development of other alternatives, some of which are touched upon by Mr. Moser. Preferred provider organizations, where the participating providers discount their charges, is perhaps the most prevalent. Hybrids have also been developed which combine features of various forms of financing health coverage. The plan described by Mr. Moser falls into that category.

With that background, I would like now to discuss what I see as likely to happen in the future. This cannot be but a fairly short-term prediction. The rate of change in medical care and financing in the United States over the past 3 or 4 years has taken place so fast that it would be foolhardy to make any predictions other than for a short period into the future.

The future is likely to see a fairly rapid spread of flexible plans - ones which are not traditional HMO's, ones which are not strictly preferred provider organizations, and which, in fact, combine features from several different versions. Members will be able to move from one type of third party financing to another. The employee, however, will have financial incentives for obtaining medical care in the tightly controlled organizations such as HMO's. This can be accomplished by changing the percentage of the costs which are paid by the various plans available to the member.

We think that the percentage of HMO's of the clinic model will decrease rapidly. We are seeing many of those plans add networks of physicians who practice in their own offices as providers for the plan. Ultimately, the role of clinics in this kind of plan may turn out to be that of providing speciality care upon referral from the primary care physicians who are practicing in their own offices.

We also are likely to see greater agreement upon what patterns of medical care treatment are acceptable for various medical conditions. Mr. Salisbury writes about some of the studies which show wide variations in frequency of surgical procedures by geographical area. These studies have focused on a greater need to adopt standards of acceptable medical practice and unnecessary medical care. HMO's and the other alternate delivery systems are likely to place an important role in influencing the adoption of such standards. 
It is becoming increasingly difficult to determine who is a health insurer. Through the 1960 's and early 1970's most insurance was provided by insurance companies; Blue Cross Blue Shield organizations, which are like insurance companies limited to health care; and a few HMO's. Now, health insurance or third party payment for health care is covered in many other ways.

We have seen the development of chains of HMO's which operate a number of HMO's for profit. Not infrequently, chains are purchasing existing HMO's which operate in only one metropolitan area. We are also seeing the purchase of smaller HMO chains by larger ones. Companies which are not insurers are also beginning to act like insurers making third party payments for medical care. Several of our large hospital organizations have gotten into this kind of arrangement. They have, however, pulled back from their positions because of concern about the profitability of the hospital organization itself and their ability to provide third party payment plans profitably.

Frequently, employers have set up their own plans without insurance and are acting as third party payers. We believe this will become less common as it becomes apparent that size is a critical factor in negotiating and developing the most favorable financial arrangements for insuring health care costs. In addition, we are seeing combinations of various kinds of organizations being formed to address the changing health insurance needs. One of our largest insurance companies, Equitable, and the largest hospital chain, Hospital Corporation of America, have formed a joint venture for this purpose. Other insurers have purchased HMO's or have developed loose associations with them in order to complete in the changing health insurance business. We expect to see more of these changes from previous roles and more joint ventures.

We believe that health care in the future will increasingly involve national organizations, but the health care itself must be provided at the local level with the national organizations providing administration, financing, and coordination. We believe that many large employers with employees scattered throughout the United States or a large geographic area, such as Southwestern Bell, will want to be able to deal with one or a limited number of organizations in arranging the financing of health care for their employees. This desire is likely to lead to the expansion of the role which large insurers such as Prudential or large HMO chains play in the health care field. A number of smaller insurers have begun to realize that they don't have the resources to develop the plans necessary to compete in the future.

Another area which is just now beginning to receive considerable attention is the evaluation of quality of medical care. Medical care quality really has not been measured in any useful quantitative way under the traditional fee-for-service provision of health care. A few HMO's and a few large employers are now addressing the question of developing meaningful measurements of quality which will permit the relative quality of different HMO's or other alternate systems to be compared. Today, the primary interest of employers in choosing a particular HMO or other system is cost. Within five years we believe that quality will be at least equal to price in the factors determining what health insurance mechanisms are chosen for the employees of many employers. 
I don't want to close these comments without making it clear that there are some real problems in health care in the United States. I believe that the most important problem is the increasing number of individuals who are not insured by either private plans or by the government. As Mr. Salisbury underlines, 37 million Americans, about $17 \%$ of the population under 65, do not have private insurance coverage or coverage from governmental sources. Two-thirds of these individuals are employed or are dependents of employed workers. There is increasing discussion at the Federal level about ways in which to provide coverage for these uninsured individuals. The most likely change is to require employers to provide a minimum level of benefits.

A second major concern is meeting the cost for long term care for the aged. We have a rapidly growing population at age 80 and over, about $25 \%$ of whom will require nursing home care. Today, the coverage for such care is almost entirely limited to that which is provided by the Medicaid program, our program for the poor. This area, too, is receiving considerable attention from government, insurers, and employers. However, the costs which are involved are enormous and are likely to expand greately. The solution must involve government and is likely to involve the private sector. Insurers can play an important role, but the coverage that they write is not likely to have a large effect until 20 or more years from now. This is because the coverage is expensive and funds must be accumulated starting at younger ages in order to pre-fund the cost of coverage at age 80 and over.

The only prediction about the future of the insurance company role in healthcare about which I can be sure is that the future will be quite different from today. We have had a rapid rate of change and that will undoubtedly continue. We can be confident that the future will include a significant role for insurers, but the role will increasingly be different from that of today. 